\title{
La sénescence cellulaire
}

\author{
Un nouveau mythe \\ de Janus?
}

> La sénescence cellulaire entraîne l'arrêt irréversible de la prolifération en réponse à divers stress génotoxiques ou stimulus inappropriés. Cet arrêt est sous le contrôle d'un réseau complexe de signalisation inhibant certains régulateurs du cycle cellulaire, comme les kinases dépendantes des cyclines (CDK). La pérennisation de cet arrêt et l'induction de la sénescence requièrent l'action de plusieurs suppresseurs de tumeurs, dont p53, pRb et $p 16^{\text {Ink4a }}$. Outre d'importants changements morphologiques et métaboliques, des altérations de la chromatine et de l'expression génique caractérisent également la sénescence. Par ailleurs, les cellules sénescentes synthétisent un ensemble de cytokines et chimiokines désigné sous le terme de senescence-associated secretory phenotype (SASP), qui favorise l'inflammation et peut modifier de façon drastique le tissu environnant. Bien qu'elle favorise la cicatrisation et s'érige en barrière oncosuppressive, la sénescence, à l'image du dieu romain Janus, offre un second visage, moins bénéfique, puisqu'elle contribue au vieillissement et aux pathologies qui lui sont associées, comme le cancer. <

\section{Sénescence cellulaire - \\ l'histoire d'un phénotype complexe}

La sénescence cellulaire a été originellement décrite par L. Hayflick qui a découvert que les cellules somatiques cessent de proliférer après un nombre précis de divisions en culture, comptabilisées selon lui par une horloge mitotique. Lorsque le nombre maximal est atteint, des signaux qui bloquent la prolifération sont alors déclenchés [1]. Contrairement à la quiescence un arrêt réversible du cycle cellulaire -, la sénescence induit un arrêt essentiellement irréversible du cycle,

Selon la mythologie romaine, Janus est le dieu à une tête mais deux visages opposés. Il est le gardien des passages et des croisements, divinité du changement et de la transition, et le symbole de la volte-face.
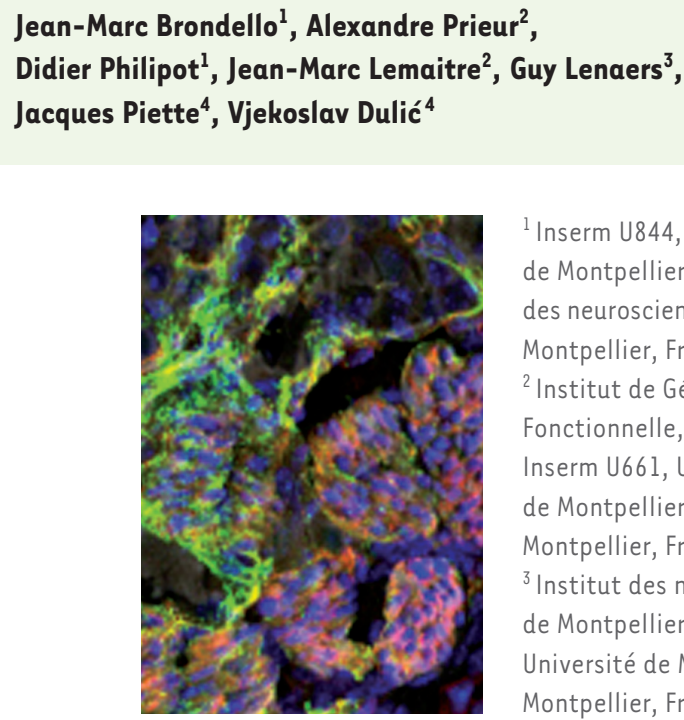

${ }^{1}$ Inserm U844, Université de Montpellier Sud, Institut des neurosciences de Montpellier, Montpellier, France;

${ }^{2}$ Institut de Génomique Fonctionnelle, CNRS UMR5203 Inserm U661, Université de Montpellier Sud, Montpellier, France ; ${ }^{3}$ Institut des neurosciences de Montpellier, Inserm U1050, Université de Montpellier Sud, Montpellier, France; ${ }^{4}$ Institut de Génétique Moléculaire de Montpellier, UMR5535, Université de Montpellier Sud, Montpellier, France. vjekoslav.dulic@igmm.cnrs.fr jacques.piette@crbm.cnrs.fr

L. Hayflick a associé la notion sénescence à celles de cancer et de vieillissement : notion de cancer car, pour échapper à la sénescence, les cellules doivent acquérir certaines caractéristiques des cellules cancéreuses; notion de vieillissement car l'accumulation de cellules sénescentes contribue au vieillissement global de l'organisme [1]. Ses hypothèses étaient fondamentalement justes, néanmoins la nature du « compteur mitotique » et des mécanismes moléculaires qui expliquent les liens entre sénescence, cancer et vieillissement ne furent découverts que récemment.

Très tôt, sur la base de considérations théoriques, A. Olovnikov avait proposé que le « compteur » suggéré par L. Hayflick pouvait être le raccourcissement des télomères, structures nucléoprotéiques protégeant les extrémités des chromosomes [3]. En effet, au fil des divisions et de la réplication des chromosomes, les télomères des cellules somatiques raccourcissent de plus en plus. Il a donc été proposé que cette érosion des télomères, reconnue par les cellules comme de I'ADN endommagé, pourrait activer la réponse au stress génotoxique, qui est identique à celle qu'induisent les rayonnements ionisants et conduit à l'arrêt de la division cellulaire, d'où le nom de «sénescence réplicative »[4]. Cette hypothèse a été confirmée par des travaux ultérieurs [5]. La sénescence peut également être déclenchée par des lésions irréparables de l'ADN, par le stress oxydatif qui survient dans des conditions de culture cellulaire non adaptées, les dysfonctions mitochondriales (voir Encadré 1), ainsi que par l'expression 


\section{Mitochondries et sénescence}

Le rôle des mitochondries dans le vieillissement cellulaire est reconnu depuis longtemps. En produisant l'énergie cellulaire sous forme d'ATP, elles génèrent aussi des espèces réactives oxygénés ( $(R 0)$ qui induisent des dommages macromoléculaires, dont l'accumulation perturbe l'ensemble du métabolisme cellulaire et l'intégrité du matériel génétique [47], deux stimulus clés de la sénescence (Figure 1). Récemment, la dynamique membranaire mitochondriale a aussi été impliquée dans la sénescence, parce qu'elle est indispensable à la mitophagie ou à l'élimination sélective des mitochondries altérées, en lien direct avec l'autophagie. Ainsi, l'inhibition de la fission mitochondriale est associée à l'accumulation de mitochondries géantes dans les cellules sénescentes, alors que la fusion mitochondriale inhiberait la sénescence et induirait la prolifération cellulaire [48]. L'altération de l'équilibre entre fusion et fission est aussi au cœur de nombreuses maladies neurodégénératives, pour lesquelles des signes de vieillissement précoce sont rapportés [49]. Par ailleurs, l'inhibition de la mitochondriogenèse semble faire partie intégrante du programme de sénescence (Figure 3). En effet, l'activation de p53 par le dysfonctionnement des télomères réduit l'expression des protéines contrôlant l'expression des constituants de la mitochondrie et la réplication de leur ADN [50], diminuant ainsi la masse mitochondriale et l'activité respiratoire, et augmentant la production d'£RO. Une telle situation chez la souris induit une atteinte cardiaque et une altération de la gluconéogenèse, deux symptômes aussi retrouvés chez les personnes vieillissantes.

supraphysiologique d'oncogènes activés comme Myc, Ras ou Raf (Figure 1). Dans tous ces cas, nous parlons de sénescence prématurée ou sénescence induite par le stress, ce qui distingue cette sénescence de la sénescence réplicative [6].

\section{p53 et pRb, acteurs clés dans l'arrêt du cycle cellulaire conduisant à la sénescence}

Dès 1991, les expériences de Jerry Shay et Woodring Wright ont mis en évidence le rôle essentiel de $p 53$ et de la protéine de rétinoblastome (pRb) dans la sénescence [7]. Ces deux suppresseurs de tumeurs, dont les voies moléculaires sont altérées dans la quasi-totalité des cellules cancéreuses, sont des acteurs clés de la réponse au stress génotoxique et de l'arrêt de la prolifération cellulaire [8]. La sénescence est étroitement associée à l'inactivation des kinases dépendantes des cyclines (CDK), principaux régulateurs du cycle cellulaire [4]. Les CDK non seulement orchestrent le bon déroulement de la réplication de l'ADN et de la mitose, mais elles maintiennent aussi pRb dans un état inactif (phosphorylé). En présence de lésions de l'ADN ou en réponse à l'hyperactivation oncogénique, les cellules activent la réponse au stress génotoxique aboutissant à l'inactivation des CDK, ce qui bloque la progression du cycle cellulaire (Figure 1). L'irréversibilité de cette inactivation est assurée par l'induction de $2^{2} 1^{\text {Wafl/cipl }}$ (p21), l'inhibiteur de CDK (CKI) dont l'expression est régulée par p53 [4, 8]. De plus, p2l prévient l'inactivation (par phosphorylation) de pRb. pRb active (non phosphorylée) séquestre les facteurs de transcription de la famille $\varepsilon 2 F$ et réprime les gènes contrôlant la division cellulaire, ce qui explique l'arrêt irréversible du cycle cellulaire associé à la sénescence non seulement en phase G1 mais également en phase G2 [9] (Figure 1). Ainsi, l'axe p21-pRb joue un rôle crucial dans l'induction de la sénescence et, comme le montrent des résultats récents, l'absence de $\mathrm{Rb}$ peut conduire à une instabilité génétique [10]. Comme nous le verrons plus loin, en s'associant à $p R b / \varepsilon 2 F$, la protéine $P M L$ (promyelocytic leukemia protein) contribue de façon importante à cet arrêt [11] (Figures l et 2).

Un autre acteur important dans l'activation de pRb lors de la sénescence est $p 16^{\operatorname{lnk4A}}$ ( $p 16$ ), un inhibiteur des kinases CDK4/6 (Figure 1) qui, à l'instar de la $\beta$-galactosidase, est un marqueur bona fide de la sénescence in vitro et in vivo $[12,13]$. La voie pl6-CDK4/6 est dérégulée dans un grand nombre de cancers soulignant son importance dans la barrière antitumorale [14]. Cependant, la contribution de pl6 à l'arrêt du cycle cellulaire reste incertaine [15] et les mécanismes de son activation au cours de la sénescence ne sont pas complètement établis. Le gène codant pour ARF, un autre suppresseur de tumeur impliqué lui aussi dans la sénescence prématurée chez la souris (Figure 1), fait partie du même locus génétique INK4a/ARF (INK4/ARF). $A R F$, comme p16, est inactif dans les cellules en prolifération, suggérant une possible corégulation des gènes du locus [8]. En effet, des résultats récents montrent que la répression épigénétique de ce locus par les protéines du groupe Polycomb ( $\mathrm{PcG}$ ) dont BMIl, est levée en réponse à un stress oncogénique [16] (Figure 1). De même, l'expression protéique de pl6 et BMIl est aussi régulée par des microARN ( $\mathrm{miR}$ ) dont le rôle dans la sénescence pourrait être essentiel [17] (Encadré 2).

\section{Les réarrangements de la chromatine associés à la sénescence}

Contrairement aux cellules quiescentes, des changements importants de la chromatine accompagnent l'arrêt du cycle cellulaire associé à la sénescence. La structure chromatinienne de certains fibroblastes humains sénescents est modifiée de façon très importante, et on observe des foyers nucléaires qui contiennent de I'hétérochromatine facultative appelés SAHF (senescence-associated heterochromatic foci, voir Glossaire) (Figures $I$ et 2) [18]. Selon le modèle actuel, cette réorganisation globale de la chromatine, qui est dépendante de $p R b$, jouerait un rôle essentiel dans l'établissement et la pérennisation (irréversibilité) de l'état sénescent [16]. Les événements moléculaires et les divers acteurs du métabolisme de la chromatine 


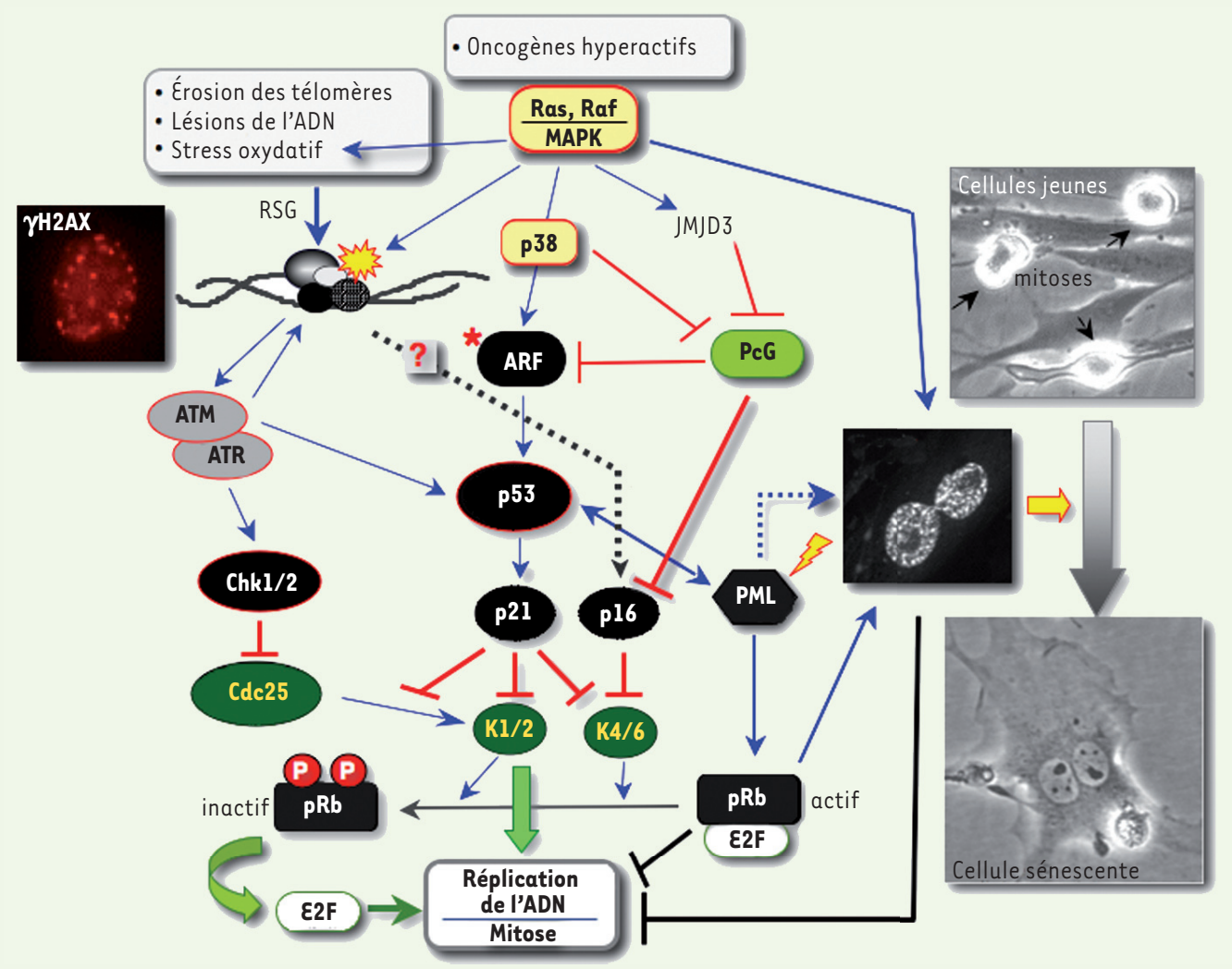

Figure 1. Mécanismes moléculaires menant à l'arrêt irréversible de la division cellulaire. Les kinases ATM/ATR (voir Glossaire) sont activées en réponse au stress génotoxique (RSG) et recrutées sur les lésions (révélées par les foyers $\gamma H 2 A X$ : voir insert) avec les systèmes de réparation de I'ADN. ATM/ATR activent les kinases CHK1 et CHK2 qui, en inhibant les phosphatases de la famille Cdc25, bloquent l'activation de CDK1 (K1) et CDK2 (K2) contrôlant la réplication de l'ADN et la mitose. Cette voie conduit à l'arrêt transitoire du cycle cellulaire. En parallèle, ATM via p53 induit p2 $1^{\text {Wafl/Cipl }}$ (p21), un inhibiteur de CDK (CKI) qui bloque la réplication et la mitose en inactivant CDK1 et CDK2 et empêche l'inactivation de pRb, ce qui assure l'irréversibilité de l'arrêt. En coopérant avec la protéine PML (voir Glossaire), pRb déclenche la sénescence en réprimant les gènes impliqués dans la division cellulaire de façon durable, en partie via l'induction des SAHF (voir Glossaire). La sénescence prématurée est également provoquée par l'expression supraphysiologique de plusieurs oncogènes qui, en activant les kinases de la voie MAPK (dont $p 38^{\text {MAPK }}$ ), stimulent la RSG et, en parallèle, stabilisent p53 via l'induction de ARF (voir Glossaire). Il faut noter que le rôle de ARF dans la sénescence n'a été démontré que dans le modèle murin (* rouge). Chez l'homme, l'hyperactivation des oncogènes induit aussi pl6 Ink4A (pl6) qui inhibe CDK4 et CDK6 (K4/K6) dont la cible principale est pRb. pl6 est également impliquée dans la sénescence réplicative et dans la formation des SAHF, mais les mécanismes contrôlant son induction ne sont pas complètement élucidés. II a été suggéré que le stress oncogénique, en induisant l'histone déméthylase JMJD3, lève la répression du locus génétique INK4A-ARF par les complexes du groupe Polycomb (PcG).

impliqués dans la formation des SAHF ne sont toujours pas complètement élucidés. Néanmoins, sur la base des données de la littérature $[18,19]$ il est possible de proposer un modèle qui pourrait expliquer, en partie au moins, l'intervention séquentielle de différentes protéines dans la mise en place des SAHF et leur rôle probable dans la répression stable des gènes contrôlant le cycle cellulaire (Figure 2).

Quel est le rapport entre les SAHF et les corps nucléaires formés par la protéine PML (promyelocytic leukemia protein), également impliqués dans la sénescence [18-20] ? Les travaux récents montrent que PML aurait un rôle important aux stades précoces de la répression des gènes cibles d'E2F en séquestrant les complexes pRb/દ2F associés à leur promoteur dans les corps nucléaires PML (PML bodies ou corps nucléaires)
[11] $(\rightarrow$ ) (Figure 2). De façon intéressante, bien que les corps nucléaires PML participent à la formation des SAHF, ces deux structures sont séparées aux stades plus tardifs de la sénescence, suggérant qu'elles exercent des fonctions différentes [11]. En effet, les SAHF ne sont pas détectés dans tous les types de cellules et pourraient ne pas être associés à toutes les formes de sénescence $[18,21]$. En accord avec ces observations, des résultats récents impliquent plutôt le stress oncogénique et l'induction de pl6 dans leur formation [22]. De plus, le rôle principal des SAHF ne serait 


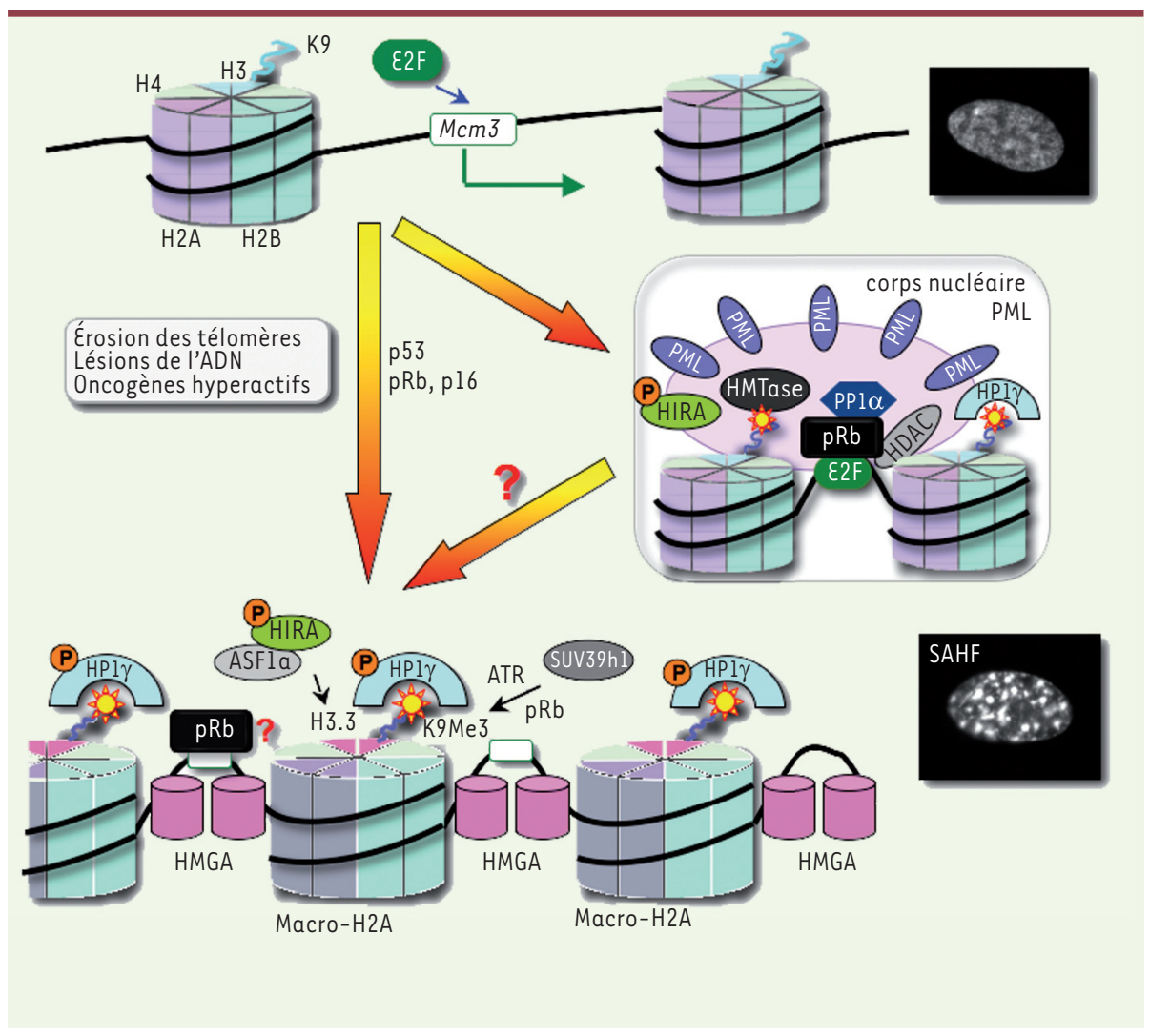

Figure 2. Les réarrangements de la chromatine associés à la sénescence : SAHF versus corps nucléaires PML. Le modèle actuel montrant la succession des événements impliqués dans la formation des SAHF au cours de la sénescence. (1) En réponse au stress génotoxique ou oncogénique, $\mathrm{pRb}$ est activée et inhibe $\varepsilon 2 F$. (2) En coopérant avec pRb, les chaperons HIRA et ASFla remplacent I'histone $\mathrm{H} 3$ par le variant H3.3 menant à la condensation de la chromatine. (3) En parallèle, les protéines chromatiniennes non-histones HMGAl/2 remplacent l'histone $\mathrm{Hl}$ linker et relient deux nucléosomes adjacents. (4) L'histone H2A est remplacée par le variant macro-H2A, un répresseur transcriptionnel, suivi par I'histone méthyltransférase SUV39hl recrutée par pRb. (5) La triméthylation de la lysine 9 (K9Me3) de l'histone $H 3$, qui est dépendante d'ATR, est reconnue par $\mathrm{HPl} \gamma$, impliquée dans la formation d'hétérochromatine. Ces modifications conduiraient à la répression stable de la transcription des gènes cibles des facteurs $22 F$ (comme Mcm3) contrôlant la réplication de I'ADN. Bien que pRb soit requis pour leur formation, sa présence dans les SAHF n'est pas complètement établie. Selon des résultats récents, dans les étapes précoces, le complexe pRB/E2F associé au promoteur est séquestré dans les structures appelées corps nucléaires PML (PML bodies) [11]. En plus des protéines hétérochromatiennes (HPl $\gamma$ ) et HDAC (histone déacétylase) qui répriment l'expression des cibles d'દ2F, les corps PML contiennent la phosphatase $1 \alpha(\mathrm{PPl} \alpha)$ qui maintiendrait pRb dans un état actif, et le chaperon HIRA qui interagit avec les histones méthyltransférases (HMTase). Les corps PML auraient également un rôle dans la formation des SAHF comme sites de «nucléation ». Les images présentent le noyau d'une cellule en prolifération (en haut) et d'une cellule sénescente contenant des SAHF (marquage par le Hoechst) (en bas).

pas de bloquer le cycle cellulaire, comme le suggèrent des résultats qui montrent que la présence des SAHF n'est pas suffisante pour arrêter la prolifération en l'absence de la voie ATM (ataxia telangiectasia mutated)-p53 et que plusieurs cellules cancéreuses surexpriment certaines composantes de SAHF [22]. À l'issue de ces travaux, on peut envisager une hypothèse selon laquelle des modifications plus locales de la chromatine (en rapport avec les corps nucléaires PML ?) seraient impliquées dans la répression des gènes qui contrôlent le cycle cellulaire tandis que le rôle de SAHF serait plutôt de maintenir l'arrêt et/ou de supprimer la réponse au stress génotoxique.

$\varepsilon$ n accord avec cette hypothèse, il a été montré que l'inhibition de la p300 histone-acétyltransférase (p300 HAT) peut bloquer le cycle cellulaire en phase G2 en induisant la formation de SAHF et la sénescence, et ce en l'absence des voies p53/p2l et pRb/p16 [23]. La progression dans la phase $S$ du cycle et la formation des SAHF sont, dans ce cas, essentielles pour l'arrêt en sénescence. Ces résultats indiquent qu'il est donc possible d'induire la sénescence indépendamment d'une activation de la réponse au stress génotoxique en agissant directement sur un changement d'organisation de la chromatine.

\section{Altération du métabolisme dans la sénescence : sécrétome et autophagie}

Les cellules sénescentes ne sont pas uniquement des cellules qui cessent de se diviser. Elles modifient aussi activement leur environnement en sécrétant des cytokines (IL[interleukine]-6/8), des chémokines, des facteurs de croissance ainsi que des protéases de la matrice extracellulaire. Ce sécrétome spécifique (ou SASP, senescence-associated secretory phenotype) joue un rôle important dans la réponse inflammatoire, dans le remodelage de la formation de la matrice extracellulaire et le maintien de l'état sénescent [6, 24] (Figure 3). Ceci est illustré par le fait que l'inhibition de I'IL-6 ou la déplétion du récepteur de I'IL-8 (IL8RB) dans des cellules sénescentes provoque un effondrement global du réseau inflammatoire et supprime la sénescence $[25,26]$. Ainsi, le sécrétome des cellules sénescentes agit de façon autocrine pour renforçer 


\section{MicroARN et sénescence}

Les microARN (miR) sont des petits ARN non codants qui répriment la traduction de leurs $A R N m$ cibles. Le lien entre sénescence et miR a été montré dans le modèle cellulaire murin, où l'ablation de Dicer, une ribonucléase clé de la biogenèse des miR, provoque un phénotype sénescent dépendant de $p 53$ in vitro et in vivo [46]. Le rôle majeur des miR, du moins ceux qui sont modifiés par Dicer, serait ainsi de prévenir la sénescence en ciblant différents acteurs clés (Figure 1). L'exemple le plus pertinent est celui du miR-24, qui cible $p 16^{\operatorname{lnk} 4 A}$ et qui est réprimé au cours de la sénescence [17]. Néanmoins, la surexpression de certains autres miR peut également induire la sénescence. Par exemple, miR$128 \mathrm{a}$, en réprimant la protéine polycomb Bmil (de la famille des polycomb, PcG) (Figure 1), permet l'expression du locus INK4A/ARF, élément clé du processus sénescent [17]. Plusieurs études ont essayé d'établir un catalogue de miR impliqués dans la sénescence. Ainsi, certains miR sont retrouvés systématiquement, comme les membres de la famille des miR-34, qui ciblent des régulateurs du cycle cellulaire ou du stress oxydatif. Cependant, comme miR-34 est induit par p53, il est possible que certains de ces miR ne soient pas à l'origine de l'arrêt prolifératif, mais en soient plutôt la conséquence. Leur rôle serait plutôt d'assurer son irréversibilité ou de réguler le sécrétome (le SASP) (Figure 3) comme le fait miRl46a/b [17].

l'état sénescent, et de façon paracrine, via la stimulation de la réponse inflammatoire, pour induire la dégénérescence tissulaire (voir plus loin) (Figure 3).

D'autre part, la sénescence a été récemment associée à l'autophagie, un processus multifonctionnel qui, en dégradant les protéines et organites, permet le recyclage des constituants défectueux de la cellule. En plus de son rôle dans le maintien de l'homéostasie métabolique, l'autophagie augmenterait la réponse au stress en contribuant à l'arrêt de la division cellulaire et en participant à la survie des cellules sénescentes [27]. L'autophagie pourrait ainsi être un effecteur de la sénescence, car elle est requise pour la sécrétion de l'IL-6 et de l'IL-8 (Figure 3). Enfin, son activité est modifiée dans des lésions prétumorales chez l'homme ce qui lui confère un rôle physiologique probable dans ce domaine [27].

Par ailleurs, il existe un lien pertinent entre la sénescence, l'autophagie et le métabolisme via mTOR (target of rapamycin), un régulateur clé de la croissance cellulaire et un répresseur de l'autophagie (Figure 3) [28]. Selon le modèle actuel, lors d'un processus de sénescence induit par l'activation des oncogènes, la voie Ras-Raf réprime les kinases du complexe mTOR (mTORC1, mTORC2), aiguillant ainsi le métabolisme cellulaire vers l'autophagie caractéristique de l'état de sénescence [27]. De plus, comme mTOR est sous le contrôle de la voie PI3K/Akt dont l'activité est dérégulée dans de nombreuses tumeurs, son activation inopinée inhiberait l'autophagie et, par conséquent, la sénescence. Enfin, les résultats qui montrent que p53 inhibe la voie IGF/PI3K/mTOR suggèrent que p53 pourrait aussi favoriser la sénescence et contrôler le métabolisme cellulaire, en partie en inhibant la mitochondriogenèse [28] (Figure 3 et Encadré 1).
Cependant, certaines observations sont en contradiction avec cette interprétation. Ainsi, les résultats de Blagosklonny et al. suggèrent que de faibles doses de p53 ne réprimeraient pas mTOR et que, au contraire, ce dernier serait nécessaire à la croissance cellulaire observée pendant la sénescence $[29,30]$. D'autre part, il a été montré récemment qu'en réponse au stress oncogénique, mTOR contribuerait à la sénescence en stimulant le SASP et l'autophagie limitée à un compartiment associé à l'appareil de Golgi $[30,31]$. Bien qu'en contradiction avec le modèle actuel (Figure 3), ces résultats, s'ils sont validés dans d'autres systèmes expérimentaux, pourraient expliquer le rôle délétère de mTOR dans le vieillissement [28].

\section{Sénescence cellulaire in vivo - ange ou démon?}

Les expériences menées in vitro suggèrent que la sénescence cellulaire agit comme une barrière antitumorale, et de nombreux résultats valident cette hypothèse in vivo en montrant que la sénescence prématurée, ainsi que la sénescence réplicative, possèdent un rôle oncosuppressif [32]. Par exemple, des cellules sénescentes sont observées dans des foyers de tumeurs bénignes mélanocytaires (naevus) [33] ou dans de nombreuses lésions précancéreuses [34], mais elles sont généralement absentes dans les tumeurs malignes où les voies $\mathrm{p} 53$ et $\mathrm{pRb}$ ont été inactivées [32]. En supprimant les cellules précancéreuses via le SASP, la sénescence constituerait donc un avantage essentiel pour la survie de l'individu par sa contribution à la répression des étapes initiales du développement tumoral (Figure 4). De plus, la sénescence participerait aussi à la cicatrisation, que favorisent la stimulation de l'inflammation et les facteurs de remodelage de la matrice extracellulaire [35] (Figure 4).

L'altération des voies impliquées dans la sénescence, en particulier celles de $p 53$ et de $\mathrm{pRb}$, dans de nombreuses tumeurs, a constitué un frein important à l'utilisation thérapeutique d'une induction spécifique de la sénescence dans les tumeurs. Néanmoins, des développements récents incitent à revoir cette attitude dans certains cas. D'une part, les inhibiteurs des p300 HAT pourraient être utilisés en thérapeutique pour induire la sénescence de cellules cancéreuses déficientes pour p53 et/ou pRb/pl6 [23]. D'autre part, le ciblage spécifique du suppresseur de tumeur PTEN pourrait induire la sénescence dans des tumeurs précoces ou des tumeurs où la fonction antiproliférative de p53 est préservée ou rétablie. Un point intéressant serait la possibilité d'induire la sénescence dans des cellules quiescentes, comme les cellules souches tumorales, 


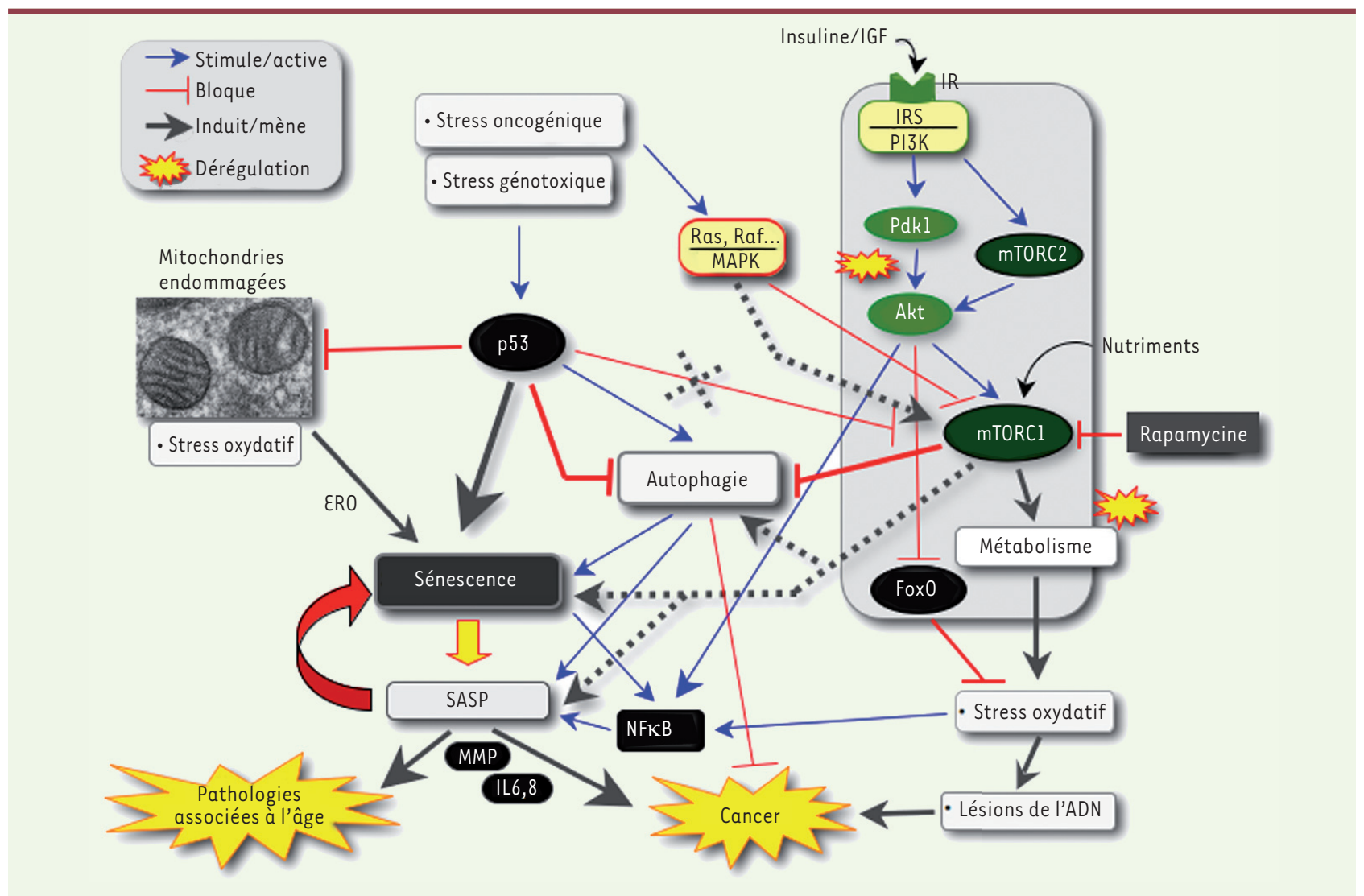

Figure 3. Rôles de l'autophagie, p53 et mTOR dans la sénescence. Le stress génotoxique (en activant p53) et le stress oncogénique induisent l'autophagie en interférant avec la voie IGF/Akt/mTOR (voir Glossaire). L'autophagie favorise la sénescence en stimulant le SASP (voir Glossaire) dans lequel NFKB joue un rôle clé. La sécrétion de cytokines (IL-6/-8) et de métalloprotéases associées à la matrice extracellulaire (MMP) provoque l'inflammation qui favorise diverses pathologies associées à l'âge (arthrose, athérosclérose et cancer). Dans certaines situations, p53 peut aussi bloquer l'autophagie. D'autre part, une stimulation excessive du métabolisme, via les mutations de la voie IGF/Akt [28], pourrait inhiber Fox0 et conduire à un stress oxydatif, également favorable à la tumorigenèse $[51,52](\rightarrow)$. P53 contribue aussi au phénotype sénescent par l'inhibition de la mitochondriogénèse, bien que la production d'espèces réactives oxygénées ( $(R 0)$ soit élevée dans les cellules sénescentes (voir Encadré 2 ). Les lignes pointillées indiquent les rôles potentiels de la voie mTOR favorisant la sénescence, suggérés par certains résultats récents [30].

$(\rightarrow)$ Voir l'article de A. Brunet, page 316 et la Nouvelle de P. Rimmelé et al., page 250 de ce numéro qui sont réfractaires à la plupart des traitements antitumoraux [36]. Enfin, l'inhibition spécifique de c-Myc ou de la sous-unité catalytique de la télomérase hTERT, peut également conduire à la sénescence de tumeurs à des stades plus avancés [36].

Les résultats de l'équipe de Judith Campisi suggèrent que paradoxalement, certains aspects de la sénescence, comme l'inflammation et la sécrétion de facteurs de remodelage de la matrice extracellulaire, pourraient également favoriser la cancérogenèse [24, 37] (Figures 3 et 4). Le fait que l'inactivation des voies $p 53$ et $p R b$, qui caractérise la plupart des tumeurs, n'interfère pas avec le sécrétome, en partie sous contrôle du facteur NF- $\kappa B$, souligne l'importance potentielle du SASP dans le développement tumoral [38]. En effet, des résultats récents dans un modèle murin montrent que l'élimination par le système immunitaire (cellules T CD4 ${ }^{+}$et macrophages) des cellules hépatiques dont la sénescence est induite par l'oncogène Nras ${ }^{G 12 V}$ est essentielle pour empêcher le développement d'un hépatocarcinome $[39,51]$. Ce processsus pourrait aussi intervenir chez l'homme. Les auteurs ont ainsi trouvé que chez les patients infectés par le virus de l'hépatite C, l'élimination des cellules sénescentes est fortement compromise par la déficience du système immunitaire en cas de coinfection par le virus VIH (virus de l'immunodéficience humaine) ou en réponse à l'administration d'immunosuppresseurs [51] $(\rightarrow)$.

L'effet délétère de la sénescence n'apparaîtrait que plus tard dans la vie, notamment parce qu'elle

$(\rightarrow)$ Voir la Nouvelle de M. Lacroix et al., page 245 de ce numéro intervient, via l'induction de pl6, dans la déplétion des cellules souches, favorisant ainsi l'altération de l'homéostasie tissulaire et conduisant au vieillissement 


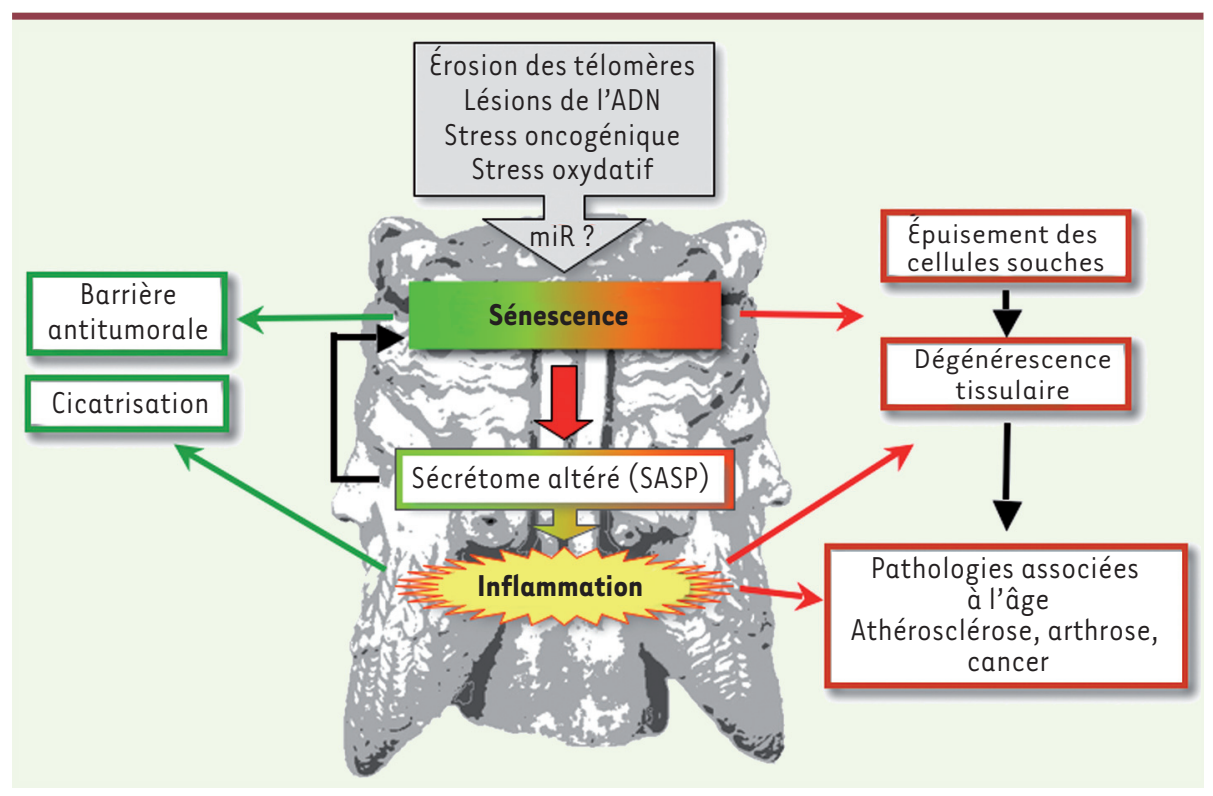

Figure 4. La sénescence cellulaire : un phénotype à deux visages opposés ? Outre les rôles apparemment «bénéfiques »(en vert), comme barrière antitumorale ou dans la cicatrisation, la sénescence cellulaire, à l'instar du dieu romain Janus, aurait un autre visage, plutôt «maléfique » (en rouge). Ainsi, en plus de l'épuisement des cellules souches, l'augmentation de cellules sénescentes dans le tissu et l'inflammation qui lui est associée présentent un danger pour les cellules avoisinantes qui peut provoquer diverses pathologies liées à l'âge comme la dégénérescence tissulaire ou le cancer.

dans des modèles murins $[32,40]$ (Figure 4) $(\rightarrow)$. De même, des cellules sénescentes associées à des foyers de lésion de l'ADN ont été observées dans la peau de babouins âgés, un argument en faveur de la réalité physiologique de ce mécanisme [41]. Ainsi, le sécrétome des cellules sénescentes, en provoquant un processus $\rightarrow$ Voir pour discussion la Nouvelle de M. Lacroix et al., page 245 de ce numéro inflammatoire, contribuerait à la dégénérescence tissulaire menant aux pathologies liées au vieillissement comme l'athérosclérose ou l'arthrose [24, 42] (Figures 3 et 4).

L'athérosclérose est caractérisée par une obstruction progressive des artères : ce processus implique les cellules endothéliales, les cellules musculaires lisses vasculaires et le système immunitaire. Une des hypothèses émises pour expliquer cette pathologie est celle d'une augmentation du nombre de cellules sénescentes, touchant d'abord la population endothéliale, puis les cellules musculaires lisses. En effet, la sécrétion massive des cytokines IL-1, IL-6 ou TGF- $\beta$ (transforming growth factor $\beta$ ) par des cellules endothéliales sénescentes pourrait attirer les cellules immunitaires comme les macrophages, celles-ci amplifiant à leur tour le signal pro-inflammatoire [24]. L'augmentation du taux de radicaux libres et des dommages à I'ADN qui en résulterait induirait un phénotype sénescent dans les cellules musculaires lisses et leur vieillissement prématuré, engendrant une perte de leur fonction et la formation progressive de plaques d'athérome (Figures 1 et 3 ).

L'arthrose, pathologie touchant principalement les genoux, les mains et les hanches, est déclenchée par une dégénérescence du cartilage associée à une augmentation de l'ossification de l'articulation. Le cartilage articulaire est un tissu organisé en plusieurs couches de chondrocytes au sein d'une matrice extracellulaire. La fonction des chondrocytes est de maintenir la structure du cartilage via la synthèse des composants de la matrice et la sécrétion d'enzymes contrôlant son renouvellement. La diminution de la fonction des chondrocytes observée au cours de la progression de l'arthrose a

\section{GLOSSAIRE}

ARF : alternative reading frame

ASFl $\alpha$ : antisilencing function $1 \alpha$

ATM : ataxia telangiectasia mutated

ATR: ATM and Rad3-related kinase

Bmil : B lymphoma Mo-MLV insertion region 1

Cde25 : cell division cycle 25 (CDK activating phosphatase)

CDK : cyclin-dependent kinase

Chk1/2 : checkpoint kinase 1/2

CKI : CDK inhibitor

عRO : espèces réactives oxygénées

FOXO : forkhead box protein 0

HAT : histone acetyl tranferase

HIRA : histone repressor $A$

HMGA : high mobility group $A$

HPI $\gamma$ : heterochromatin protein $1 \gamma$

IGF : insulin-like growth factors

IRS : insulin receptor substrate

INK4A : inhibitor of Cdk4 A

MAPK : mitogen-activated protein kinase

mTOR : mammalian target of rapamycin

NFKB : nuclear factor $\kappa B$

TORC1/2 : TOR complex 1 ou 2

PcG : Polycomb group

PDK1 : 3-phosphoinositide-dependent kinase 1

PI3K : phosphoinositide 3 kinase

PML : promyelocytic leukemia protein

PTEN : phosphatase and tensin homologue

$\mathbf{p R b}$ : protéine de rétinoblastome

SAHF : senescence-associated heterochromatin foci

SASP : senescence-associated secretory phenotype

TGF $\beta$ : transforming growth factor $\beta$ 
été attribuée à leur sénescence prématurée et à la sécrétion des molécules pro-inflammatoires et des métalloprotéases associées à la matrice extracellulaire (Figure 3). Cette sénescence prématurée serait due aux stress mécaniques répétés et à l'accumulation des radicaux libres, affectant l'homéostasie du tissu et engendrant une dégradation progressive du cartilage [43].

Pour conclure, il faut signaler que si ces liens complexes entre sénescence, cancer et vieillissement ont été identifiés expérimentalement dans de nombreux modèles animaux, ils demeurent difficiles à démontrer formellement chez l'homme.

\section{Épilogue}

Comme le dieu romain Janus, la sénescence cellulaire aurait donc deux visages (Figure 4). L'un, avenant, contribue à la suppression tumorale et à la cicatrisation, l'autre, plus sournois, participe au vieillissement et aux pathologies associées à l'âge avancé [24, 44]. Cet aspect sombre de la sénescence ne serait pas soumis à une forte pression de sélection au cours de l'évolution, car il n'affecte que des individus qui ont dépassé l'âge de se reproduire. L'ambivalence de la sénescence est bien illustrée par un de ses principaux acteurs, le CKI pl6 : alors que sa répression caractérise de nombreuses tumeurs, son invalidation permet aussi d'accroître le compartiment des cellules souches tissulaires et donc potentiellement la durée de vie. De plus, l'élimination des cellules sénescentes qui surexpriment pl6 supprime fortement les pathologies associées au vieillissement $[13,51]$. II a d'ailleurs été démontré qu'il était possible de reprogrammer in vitro les cellules sénescentes humaines et de les « rajeunir »[45]. Une thérapie ciblant les cellules sénescentes pourrait donc potentiellement diminuer les effets néfastes du vieillissement. Mais est-il possible de combattre spécifiquement le vieillissement sans augmenter le risque de développement tumoral ? C'est sans aucun doute un défi qu'il faudra relever par une meilleure compréhension de l'ensemble des mécanismes qui induisent et caractérisent la sénescence. Une approche séduisante serait de s'attaquer plus spécifiquement à certaines de ses caractéristiques, comme le SASP (sécrétome altéré) qui, en stimulant l'inflammation, favoriserait le développement de certaines pathologies (Figures 3 et 4). Néanmoins, les liens fonctionnels étroits entre les différentes manifestations de la sénescence pourraient sérieusement compliquer cette démarche. $\diamond$

\section{SUMMARY}

Cellular senescence and the myth of Janus

Cellular senescence is, essentially, a permanent proliferation arrest induced by various cellular stresses or inappropriate stimuli. This arrest, which is associated with dramatic changes in cell morphology, metabolism and gene expression, involves a complex signalling network aiming at stable inactivation of CDKs, major cell cycle regulators. Notably, several tumour suppressors, such as $\mathrm{p} 53$, pRb or p16 ${ }^{\operatorname{lnk} 4 a}$, play key roles both in the initiation of the senescence program and in its maintenance, which often involves epigenetic changes. While having widely recognized roles in tumour suppression and wound healing, senescence, like the roman god Janus, recently revealed another darker face. Mostly due to altered secretion phenotype favouring inflammation, senescent cells strongly influence surrounding tissue contributing to the development of age-related pathologies, including cancer. $\diamond$

\section{CONFLIT D'INTÉRÊTS}

Les auteurs déclarent n'avoir aucun conflit d'intérêts concernant les données publiées dans cet article.

\section{RÉFÉRENCES}

1. Hayflick L. The limited in vitro lifetime of human diploid cell strains. Exp Cell Res $1965 ; 37: 614-36$.

2. Gire V. La sénescence : une barrière télomérique à l'immortalité ou une réponse cellulaire aux stress physiologiques? Med Sci (Paris) 2005; 21: 491-7.

3. Olovnikov AM. A theory of marginotomy. The incomplete copying of template margin in enzymic synthesis of polynucleotides and biological significance of the phenomenon.J Theor Biol 1973; 41 : 181-90.

4. Stein GH, Dulic V. Origins of Gl arrest in senescent human fibroblasts. Bioessays $1995 ; 17: 537-43$.

5. D’Adda di Fagagna F, Reaper PM, Clay-Farrace L, et al. A DNA damage checkpoint response in telomere-initiated senescence. Nature 2003; 426 : 194-8.

6. Bischof 0 , Dejean A, Pineau P. Une revue de la sénescence cellulaire - Ami ou ennemi de la promotion tumorale? Med Sci (Paris) $2009 ; 25: 153-60$.

7. Shay JW, Pereira-Smith OM, Wright WE. A role for both RB and $p 53$ in the regulation of human cellular senescence. Exp Cell Res 1991; 196 : 33-9.

8. Polager S, Ginsberg D. p53 and E2f: partners in life and death. Nat Rev Cancer $2009 ; 9: 738-48$.

9. Baus F, Gire V, Fisher D, et al. Permanent cell cycle exit in G2 phase after DNA damage in normal human fibroblasts. EMBO J 2003 ; 22 : 3992-4002.

10. Chicas $A$, Wang $X$, Zhang $C$, et al. Dissecting the unique role of the retinoblastoma tumor suppressor during cellular senescence. Cancer Cell $2010 ; 17: 376-87$.

11. Vernier M, Bourdeau V, Gaumont-Leclerc MF, et al. Regulation of \&2Fs and senescence by PML nuclear bodies. Genes Dev $2011 ; 25: 41-50$.

12. Krishnamurthy J, Torrice C, Ramsey MR, et al. Ink4a/Arf expression is a biomarker of aging. J Clin Invest $2004 ; 114$ : 1299-307.

13. Baker DJ, Wijshake T, Tchkonia T, et al. Clearance of pl6(Ink4a)-positive senescent cells delays ageing-associated disorders. Nature $2011 ; 479$ : 232-6.

14. Gil J, Peters G. Regulation of the INK4b-ARF-INK4a tumour suppressor locus: all for one or one for all. Nat Rev Mol Cell Biol $2006 ; 7$ : 667-77.

15. Herbig U, Jobling WA, Chen BP, et al. Telomere shortening triggers senescence of human cells through a pathway involving ATM, p53, and p21(CIP1), but not pl6(INK4a). Mol Cell $2004 ; 14: 501-13$.

16. Kuilman T, Michaloglou C, Mooi WJ, Peeper DS. The essence of senescence. Genes Dev 2010 ; $24: 2463-79$.

17. Gorospe M, Abdelmohsen K. Microregulators come of age in senescence. Trends Genet $2011 ; 27: 233-41$.

18. Narita $M$, Nunez $S$, Heard $\varepsilon$, et al. Rb-mediated heterochromatin formation and silencing of $\varepsilon 2 F$ target genes during cellular senescence. Cell 2003 ; $113: 703-16$.

19. Adams PD. Remodeling of chromatin structure in senescent cells and its potential impact on tumor suppression and aging. Gene $2007 ; 397: 84-93$.

20. Ferbeyre G, de Stanchina $\varepsilon$, Querido $\varepsilon$, et al. PML is induced by oncogenic ras and promotes premature senescence. Genes Dev $2000 ; 14$ : 2015-27.

21. Kennedy AL, McBryan T, Enders GH, et al. Senescent mouse cells fail to overtly regulate the HIRA histone chaperone and do not form robust senescence associated heterochromatin foci. Cell Div $2010 ; 5: 16$.

22. Di Micco R, Sulli G, Dobreva M, et al. Interplay between oncogene-induced DNA damage response and heterochromatin in senescence and cancer. Nat Cell Biol 2011; 13 : 292-302.

23. Prieur A, Besnard E, Babled A, Lemaitre JM. p53 and p16(INK4A) independent induction of senescence by chromatin-dependent alteration of $S$-phase progression. Nat Commun $2011 ; 2: 473$. 


\section{RÉFÉRENCES}

24. Rodier F, Campisi J. Four faces of cellular senescence. J Cell Biol 2011 ; 192 : 547-56.

25. Kuilman T, Michaloglou C, Vredeveld LC, et al. Oncogene-induced senescence relayed by an interleukin-dependent inflammatory network. Cell $2008 ; 133$ : 1019-31.

26. Acosta JC, O'Loghlen A, Banito $A$, et al. Chemokine signaling via the CXCR2 receptor reinforces senescence. Cell $2008 ; 133: 1006-18$

27. Young AR, Narita M. Connecting autophagy to senescence in pathophysiology. Curr Opin Cell Biol $2010 ; 22: 234-40$.

28. Zoncu R, Efeyan A, Sabatini DM. mTOR: from growth signal integration to cancer, diabetes and ageing. Nat Rev Mol Cell Biol $2011 ; 12$ : 21-35.

29. Demidenko ZN, Korotchkina LG, Gudkov AV, Blagosklonny MV. Paradoxical suppression of cellular senescence by p53. Proc Natl Acad Sci USA 2010 ; 107 : 9660-4.

30. Pani G. From growing to secreting: New roles for mTOR in aging cells. Cell Cycle $2011 ; 10: 2450-3$.

31. Narita M, Young AR, Arakawa S, et al. Spatial coupling of mTOR and autophagy augments secretory phenotypes. Science 2011 ; 332 : 966-70.

32. Collado M, Serrano M. Senescence in tumours: evidence from mice and humans. Nat Rev Cancer $2010 ; 10: 51-7$

33. Michaloglou C, Vredeveld LC, Soengas MS, et al. BRAFE600-associated senescence-like cell cycle arrest of human naevi. Nature $2005 ; 436: 720-4$.

34. Bartkova J, Rezaei N, Liontos M, et al. Oncogene-induced senescence is part of the tumorigenesis barrier imposed by DNA damage checkpoints. Nature $2006 ; 444: 633-7$.

35. Krizhanovsky V, Yon M, Dickins RA, et al. Senescence of activated stellate cells limits liver fibrosis. Cell 2008; $134: 657-67$

36. Nardella C, Clohessy JG, Alimonti A, Pandolfi PP. Pro-senescence therapy for cancer treatment. Nat Rev Cancer 2011; 11: 503-11.

37. Coppe JP, Patil CK, Rodier F, et al. Senescence-associated secretory phenotypes reveal cellnonautonomous functions of oncogenic RAS and the p53 tumor suppressor. PLoS Biol $2008 ; 6$ : 2853-68.

38. Adams PD. Healing and hurting: molecular mechanisms, functions, and pathologies of cellula senescence. Mol Cell $2009 ; 36: 2-14$.

39. Kang TW, Yevsa T, Woller $\mathrm{N}$, et al. Senescence surveillance of pre-malignant hepatocytes limits liver cancer development. Nature 2011 ; 479 : 547-51.

40. Collado M, Blasco MA, Serrano M. Cellular senescence in cancer and aging. Cell $2007 ; 130$ : 223-33.
41. Herbig U, Ferreira M, Condel L, et al. Cellular senescence in aging primates. Science $2006 ; 311: 1257$

42. Rodier F, Coppe JP, Patil CK, et al. Persistent DNA damage signalling triggers senescence-associated inflammatory cytokine secretion. Nat Cell Biol 2009; $11: 973-9$

43. Brondello JM, Philipot D, Djouad F, et al. Cellular senescence is a common characteristic shared by preneoplasic and osteo-arthritic tissue. Open Rheumatol J $2010 ; 4: 10-4$

44. Serrano M, Blasco MA. Cancer and ageing: convergent and divergent mechanisms. Nat Rev Mol Cell Biol $2007 ; 8: 715-22$

45. Lapasset L, Milhavet 0 , Prieur $A$, et al. Rejuvenating senescent and centenarian human cells by reprogramming through the pluripotent state. Genes Dev $2011 ; 25$ : 2248-53.

46. Mudhasani R, Zhu Z, Hutvagner $G$, et al. Loss of miRNA biogenesis induces p19Arf-p53 signaling and senescence in primary cells. J Cell Biol 2008; 181 1055-63

47. Passos JF, Saretzki G, von Zglinicki T. DNA damage in telomeres and mitochondria during cellular senescence: is there a connection? Nucleic Acids Res 2007 ; 35 : 7505-13.

48. Yoon YS, Yoon DS, Lim IK, et al. Formation of elongated giant mitochondria in DFO-induced cellular senescence: involvement of enhanced fusion process through modulation of Fis 1. J Cell Physiol 2006 ; 209 : 468-80.

49. Lenaers G, Amati-Bonneau P, Delettre $C$, et al. De la levure aux maladies neurodégénératives - Dix ans d'exploration des pathologies de la dynamique mitochondriale. Med Sci (Paris) $2010 ; 26: 836-41$.

50. Sahin $\varepsilon$, Colla $S$, Liesa M, et al. Telomere dysfunction induces metabolic and mitochondrial compromise. Nature 2011 ; 470 : 359-65.

51. Lacroix M, Linares L, LeCam L. Le Yin et le Yang de la sénescence : est-il possible de vieillir sans développer de cancer? Med Sci (Paris) $2012 ; 28$ $245-7$

52. Rimmelé $P$, Zhang $X$, Ghaffari $S$. Rôle des facteurs de transcription Fox 0 dans la maintenance des cellules souches. Med Sci (Paris) 2012 ; 28 : 250-4.

\section{TIRÉS À PART}

V. Dulic

\section{Collection L'Actualité Chimique-Livres}

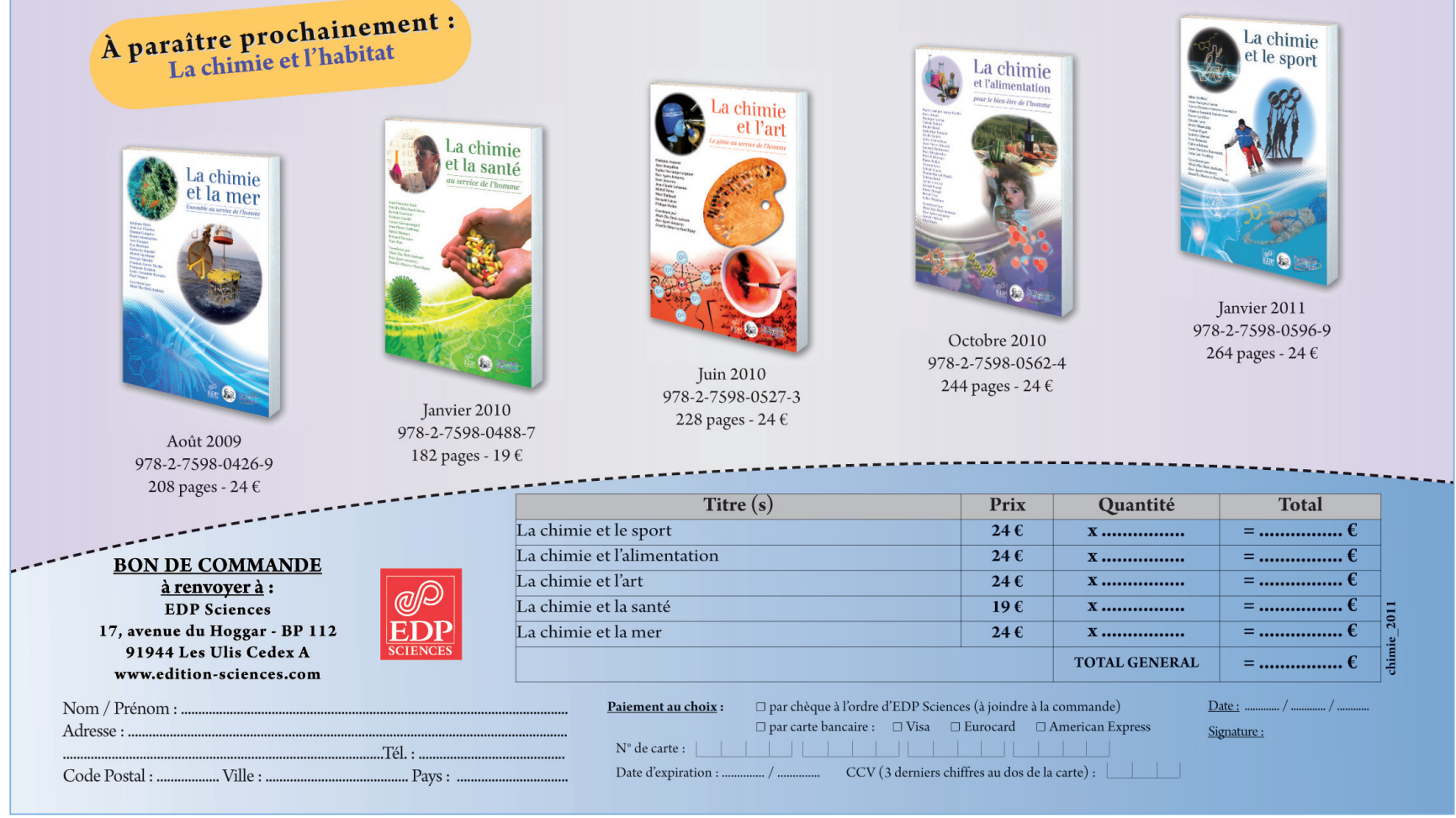

